\title{
BMJ Open Efficacy and safety of nintedanib in Japanese patients with early-stage idiopathic pulmonary fibrosis: a study protocol for an observational study
}

\author{
Noriho Sakamoto (D , , ${ }^{1}$ Naoki Hamada, ${ }^{2}$ Masaki Okamoto, ${ }^{3,4}$ Kazunori Tobino, ${ }^{5}$ \\ Hidenori Ichiyasu, ${ }^{6}$ Hiroshi Ishii, ${ }^{7}$ Kazuya Ichikado, ${ }^{8}$ Shimpei Morimoto, ${ }^{9}$ \\ Naoki Hosogaya, ${ }^{10}$ Hiroshi Mukae ${ }^{1}$
}

To cite: Sakamoto $\mathrm{N}$, Hamada N, Okamoto M, et al. Efficacy and safety of nintedanib in Japanese patients with earlystage idiopathic pulmonary fibrosis: a study protocol for an observational study. BMJ Open 2021;11:e047249. doi:10.1136/ bmjopen-2020-047249

- Prepublication history for this paper is available online. To view these files, please visit the journal online (http://dx.doi. org/10.1136/bmjopen-2020047249).

Received 26 November 2020 Accepted 02 June 2021

Check for updates

(C) Author(s) (or their employer(s)) 2021. Re-use permitted under CC BY-NC. No commercial re-use. See rights and permissions. Published by BMJ.

For numbered affiliations see end of article.

Correspondence to

Dr Noriho Sakamoto;

nsakamot@nagasaki-u.ac.jp

\section{ABSTRACT}

Introduction Idiopathic pulmonary fibrosis (IPF) is a fibrotic disease of unknown aetiology with a poor prognosis. Several clinical trials of nintedanib in patients with IPF have reported its inhibitory effect on reduced lung function, incidence of acute exacerbation of IPF and worsened health-related quality of life. Although nintedanib has a manageable safety and tolerability profile over long-term use, it was discontinued in over $20 \%$ of patients because of adverse events such as diarrhoea and liver dysfunction. This might explain why nintedanib use in patients with IPF is not widespread, especially among patients with early-stage IPF. In the present study, we aimed to clarify the efficacy, safety and tolerability of nintedanib in patients with stage I/IIIPF, based on the Japanese IPF disease severity staging classification system.

Methods and analysis This is an ongoing, prospective, multicentre observational cohort study of patients with stage I/II IPF who will start receiving nintedanib. Totally, 215 patients at 35 sites in Kyushu and Okinawa, Japan will be enrolled and followed up for 3 years. Nintedanib therapy would be initiated at the discretion of the investigator. The primary endpoint, change in forced vital capacity (FVC) at 156 weeks, will be shown as the mean change in FVC from baseline to week 156 with $95 \%$ Cls estimated using the Wald method. The safety endpointoccurrence of adverse events-will be assessed in each system organ class/preferred term.

Ethics and dissemination The study protocol and informed consent documents were approved by the Institutional Review Board at Nagasaki University Hospital (approval number 19102146) and each participating site. Written informed consent was obtained from all participants. Patient recruitment has begun. The results will be disseminated through scientific peer-reviewed publications and national and international conferences. Trial registration number UMIN000038192.

\section{INTRODUCTION}

Idiopathic pulmonary fibrosis (IPF) is a fibrotic disease of an unknown aetiology affecting the lung interstitium. Its primary pathogenesis is suspected to be chronic injury
Strengths and limitations of this study

- This is a real-world, prospective, multicentre observational cohort study.

- The results will reveal the efficacy, safety and tolerability of nintedanib in Japanese patients with earlystage idiopathic pulmonary fibrosis.

- The study is designed as a prospective, single-arm, non-randomised study.

- Participating sites are located in specific areas of Japan, which may cause some biases regarding patient background.

to the alveolar epithelial cells, which causes chronic fibrosis. IPF occurs in $50 \%-60 \%$ of patients with idiopathic interstitial pneumonia. It has a poor prognosis with a median survival time of approximately 3 years. ${ }^{12} \mathrm{~A}$ decline in pulmonary function, worsening of pulmonary symptoms and physical activity, and acute exacerbation have been identified as risk factors for the poor prognosis. ${ }^{3}$ Following a definitive diagnosis, the median survival time of patients with percentage vital capacity $(\% \mathrm{VC})$ of $>80 \%$ in pulmonary function test, in a relatively early stage, has been reported to be 62 months. Furthermore, the median survival of patients with stage I and II IPF, classified by the Japanese IPF disease severity staging classification system, is 62 and 51 months, respectively. ${ }^{4}$ These results indicate that even patients in the early stages do not have a good prognosis.

Treatments that modulate inflammation (eg, steroids and immunosuppressants) have failed or had deleterious effects in patients with IPF; currently, two medications, nintedanib and pirfenidone, have been shown to be safe and effective in the treatment of IPF. ${ }^{5}$ Several clinical trials of nintedanib in patients with IPF have reported 
its inhibitory effect on reduced lung function, incidence of acute exacerbation of IPF and worsened health-related quality of life (QOL) ${ }^{5-7}$ Pooled data from phase II and III trials have revealed that nintedanib reduced all-cause and on-treatment mortality. ${ }^{8}$ In addition, INPULSIS-ON, an open-label nintedanib extension trial, revealed the effect of nintedanib in reducing the annual rate of decline in forced vital capacity (FVC) and showed that acute exacerbation of IPF persists beyond 4 years. ${ }^{7}$ A reduction in the annual rate of FVC decline was also observed in patients with early-stage IPF. ${ }^{7}$ These results suggest that early treatment with nintedanib maintains QOL and prolongs survival, which could influence the perspective of patients with IPF. Therefore, the use of nintedanib is recommended for patients with IPF in the official American Thoracic Society/European Respiratory Society/ Japanese Respiratory Society/Latin American Thoracic Association (ATS/ERS/JRS/ALAT) clinical practice guidelines $^{3}$ and Japanese guidelines for the treatment of IPF. $^{9}$

However, adverse events, such as diarrhoea, nausea and liver dysfunction, have been attributed to nintedanib use. Although nintedanib has a manageable safety and tolerability profile for long-term use, it was discontinued in over $20 \%$ of patients because of adverse events. ${ }^{67}$ The long-term continuation rate has been reported to be significantly higher in the mild-to-moderate patient group. ${ }^{10}$ The effect of nintedanib on slowing disease progression is also sustained over a long term, and long-term treatment with nintedanib has an acceptable safety and tolerability profile in Asian patients with IPF. ${ }^{11}$ Furthermore, a subgroup analysis of the INPULSIS trials showed comparable efficacy and safety of nintedanib in Japanese patients and the overall population. ${ }^{12}$ However, no prospective study has reported the efficacy, safety and tolerability of nintedanib in Japanese patients with earlystage IPF.

The present study aimed to clarify the (1) efficacy, safety and tolerability of nintedanib over a 1-year observation period, and (2) efficacy of nintedanib over a 3-year observation period in patients with stage I/II IPF based on the Japanese IPF disease severity staging classification system. Herein, we describe the study objectives, design and endpoints.

\section{METHODS AND ANALYSIS \\ Study design}

This is an ongoing, prospective, multicentre observational cohort study. The present study has been designed in accordance with the Strengthening the Reporting of Observational Studies in Epidemiology Statement guidelines.

\section{Patient and public involvement}

Patients will not be involved in the study.

\section{Participant selection and screening}

\section{Study population}

In total, 215 participants diagnosed with IPF will be recruited from sites in Kyushu and Okinawa, Japan, from 24 October 2019. IPF will be diagnosed by pulmonary physicians in each site according to the ATS/ERS/ JRS/ALAT guidelines. ${ }^{13}$ Usual interstitial pneumonia or probable usual interstitial pneumonia pattern in highresolution CT (HRCT) will be diagnosed as IPF if surgical lung biopsy is not performed. The choice of treatment will be based on regular medical practice and at the discretion of the physician. The specific inclusion and exclusion criteria for the trial are provided below.

\section{Inclusion criteria}

1. Diagnosis of IPF according to the ATS/ERS/JRS/ ALAT guidelines. ${ }^{13}$

2. Stage I or II IPF based on the Japanese IPF disease severity classification. ${ }^{4}$

Stage I: $\mathrm{PaO}_{2}$ at rest $\geq 80 \mathrm{~mm} \mathrm{Hg}$.

Stage II: $\mathrm{PaO}_{2}$ at rest $70-80 \mathrm{~mm} \mathrm{Hg}$, and $\mathrm{SpO}_{2} \geq 90 \%$ during a 6-min walk test.

3. Patients who will start nintedanib for IPF.

4. Patients who are able and willing to provide written informed consent.

\section{Exclusion criteria}

1. Patients who have initiated therapy with any antifibrotic agent for IPF.

2. Patients treated with corticosteroids (more than a prednisolone equivalent dose, $10 \mathrm{mg} /$ day) or immunosuppressive agents.

3. Patients who are participating in any other interventional clinical trials.

4. Patients who are undergoing malignant tumour treatment.

5. Patients with a contraindication for nintedanib treatment.

\section{Data collection and management}

Patients will be included in the study following the provision of informed signed consent and if all inclusion criteria are fulfilled without meeting any of the exclusion criteria. The following data will be collected and registered during a month before starting nintedanib: sex, date of birth, height, weight, body surface area, smoking history, social history (presence or absence of dust exposure, contact with birds and occupational history), family history of interstitial pneumonia, date of IPF diagnosis, presence or absence of surgical lung biopsy, findings of bronchoalveolar lavage fluid, history of treatment for interstitial pneumonia, previous and current concomitant medications, respiratory function tests in the past 6-15 months, medical history and complications. The following physical examination results will be collected: $\mathrm{SpO}_{2}$, dyspnoea scale score (modified Medical Research Council), biological assessments (including blood count and renal and hepatic functions) and serum interstitial 
Table 1 Schedule of activities

\begin{tabular}{|c|c|c|c|c|c|c|c|c|c|c|c|c|}
\hline \multirow[b]{2}{*}{ Time } & \multirow[b]{2}{*}{ Screening } & \multicolumn{5}{|c|}{ 1-year observation period } & \multicolumn{4}{|c|}{ 3-year observation period } & \multirow{2}{*}{$\begin{array}{l}\text { Stop } \\
\text { NIN }\end{array}$} & \multirow{2}{*}{$\begin{array}{l}\text { Stop } \\
\text { study }\end{array}$} \\
\hline & & $4 W$ & $12 W$ & $24 W$ & $36 \mathrm{~W}$ & $52 W$ & $78 W$ & $104 W$ & $130 W$ & $156 \mathrm{~W}$ & & \\
\hline Consent & - & - & - & - & - & - & - & - & - & - & - & - \\
\hline NIN (dose) & - & - & $\bullet$ & - & - & ○ & - & - & - & - & $\mathrm{O}$ & O \\
\hline Symptom (mMRC) & $\bullet$ & - & - & - & - & - & - & - & - & - & $\mathrm{O}$ & O \\
\hline Routine blood tests & $\bullet$ & - & $\bullet$ & $\bullet$ & - & - & $\bullet$ & $\bullet$ & - & $\bullet$ & $\mathrm{O}$ & $\mathrm{O}$ \\
\hline Serum ILD markers & 0 & - & - & 0 & - & 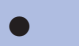 & ? & 0 & 0 & 0 & O & O \\
\hline Blood gas analysis & $\bullet$ & - & - & - & - & $\bullet$ & - & ○ & - & $\bullet$ & $\mathrm{O}$ & $\mathrm{O}$ \\
\hline Chest XP, HRCT & 0 & - & - & - & - & 0 & - & 0 & - & 0 & O & $\mathrm{O}$ \\
\hline 6MWT & $\bullet$ & - & - & - & - & $\bullet$ & - & - & - & - & $\mathrm{O}$ & $\mathrm{O}$ \\
\hline Acute exacerbation & - & $\bullet$ & $\bullet$ & - & $\bullet$ & $\bullet$ & $\bullet$ & $\bullet$ & $\bullet$ & $\bullet$ & O & $\mathrm{O}$ \\
\hline Drugs other than NIN & - & $\bullet$ & - & $\bullet$ & $\bullet$ & $\bullet$ & - & $\bullet$ & $\bullet$ & $\bullet$ & O & $\mathrm{O}$ \\
\hline Prognosis & - & - & - & - & - & - & - & - & - & $\bullet$ & - & $\bullet$ \\
\hline Adverse events & - & 0 & 0 & 0 & 0 & 0 & 0 & 0 & 0 & 0 & O & O \\
\hline
\end{tabular}

: Mandatory; O: if possible.

HRCT, high-resolution CT; ILD, interstitial lung disease; mMRC, modified Medical Research Council dyspnoea scale; 6MWT, 6 min walk test; NIN, nintedanib; PFT, pulmonary function test; UCG, ultrasound cardiography; W, week; XP, X-ray photography.

lung disease markers (Krebs von den Lungen-6 (KL-6), Surfactant Protein-D (SP-D)).$^{14}$ HRCT, pulmonary function tests, blood gas analysis and the 6-minute walk test will be performed within 3 months before inclusion in the study, and the Japanese classification of IPF disease severity and the Gender-Age-Physiology Index for IPF ${ }^{15}$ will be determined. Electrocardiography and echocardiography results will be collected if they are performed within the past 6 months.

Study treatment will be started within a month after the provision of informed signed consent, and the starting date and daily dose of nintedanib will be recorded. Follow-up data will be collected at weeks 4, 12, 24, 36, 52, 78, 104, 130 and 156. During each visit, a physical examination including $\mathrm{SpO}_{2}$ and biological assessments will be regularly performed. The occurrence of adverse and acute exacerbation events ${ }^{16}$ will be recorded at clinical visits and between visits as required to monitor treatment safety. The daily dose of nintedanib and the date of any reduction or discontinuation of nintedanib will be recorded. The reduction or discontinuation of nintedanib will be determined in each site. In addition, the results of serum interstitial lung disease markers, pulmonary function test, HRCT, 6-min walk test and blood gas analysis will be collected at each interval (table 1). The patients will be followed up until death, lung transplantation, diagnosis of non-IPF or study completion. Efficacy and safety endpoints will be collected up to 52 weeks after the start of oral administration (safety priority observation period), and an interim analysis will be conducted 52 weeks after the initiation of oral administration. To observe the longterm therapeutic effect, details will be collected during the 156 weeks after the start of oral administration (effectiveness-focused observation period). Patients will be registered using a interactive web response system and source data entry will be made in a standard case report form, with direct electronic entry using REDCap hosted by the Nagasaki University Hospital.

\section{Primary endpoint}

Change in FVC at 156 weeks.

\section{Safety endpoints}

Occurrence of adverse events in each system organ class/ preferred term (SOC/PT; MedDRA V.22.1).

\section{Secondary endpoints}

1. Time to the incidence of adverse events leading to nintedanib treatment discontinuation.

2. Time to the incidence of adverse events leading to nintedanib dose reduction.

3. Incidence of adverse events leading to nintedanib treatment discontinuation.

4. Incidence of adverse events leading to nintedanib dose reduction.

Further endpoints

1. Time to the first acute exacerbation of IPF.

2. Time to absolute $10 \%$ decline in $\% \mathrm{FVC}$ from baseline. 
3. Time to death.

4. Change in FVC from baseline at each interval.

5. Change in the diffusion capacity of carbon monoxide (DLco) from baseline at each interval.

6. Change in serum biomarkers (KL-6 and SP-D) from baseline at each interval.

7. Judgement of therapeutic efficacy at 52, 104 and 156 weeks (a change in absolute FVC of $10 \%$ or a change in absolute DLco of $15 \%$ ) according to a previous report. ${ }^{17}$

\section{Statistical analysis}

\section{Sample size calculation}

The sample size was determined from a precision analysis of the primary endpoint and aimed to assure that liver injury was observed in at least one patient. The precision analysis was conducted based on the mean change in FVC from baseline to week 192 in 138 patients obtained from the nintedanib-continuing group of a previous study. ${ }^{7}$ Using this result, we set the sample size of 44 to a $95 \%$ CI (CI.95) narrower than $15(\mathrm{~mL})$ obtained with the probability exceeding $95 \%$. Finally, we estimated the proportion of dropout patients as 24/119 based on the results of the Asian cohort in a previous study ${ }^{11}$ and set the sample size as 215 .

The sample size needed to observe liver injury was determined as follows. (1) The CI.95 of the frequency of liver injury in a patient was estimated using the Wilson score interval. The point estimate of the frequency was obtained from the Japanese package insert of Ofev (17 events in 638 patients). We obtained the lower and upper limits of the CI.95 (lower and upper control limits) as 0.0156 and 0.0451 , respectively. (2) The number of patients who exhibited liver injury was simulated from the binomial distribution with sample sizes of 150, 180, 190, 195, 200, 205, 210, 215, 220, 225, 230, 235, 240, 245, 250, 300 and 310 . The lower control limit was adopted as parameter $p$ of the binomial distribution because the purpose of this sample size assessment was to ensure the observation of the clinical course of liver injury occurring as an adverse effect of nintedanib, even if the true frequency of liver injury occurrence is extremely small within the possible range. For each sample size, 5000 observations were generated. (3) The probability $1-p_{N}(0)$ was obtained for each sample size $N$, where $p_{N}(k)$ denotes the probability mass function of the number of patients who exhibited liver injury $k$ of the $N$ patients, which was estimated from the empirical distribution of the 5000 observations. (4) Finally, the sample size for this study was determined as $n=\inf \left\{N: 1-p_{N}(0)>0.95\right\}$. We obtained $n=215$.

Enrollment will continue until at least $50 \%$ of patients with stage I IPF according to the Japanese classification of disease severity are recruited.

\section{Analysis of outcome measures}

Details of the analysis methods will be documented in the statistical analysis plan that will be signed off before database freeze with all participants' data. The primary analysis will focus on the mean change in FVC from baseline to week 156 with the CI.95 estimated using the Wald method. For the safety analysis, adverse events in each SOC/PT will be assessed. Regarding the secondary analysis, the Kaplan-Meier method will be used to estimate the survival function of time to events. A Cox proportional hazard model will be used for conducting an exploratory analysis with patient characteristics. The association of clinical factors with the occurrence of adverse events leading to treatment discontinuation or dose reduction of nintedanib will be analysed using logistic regression models. The data collected in a time-series manner will be analysed using mixed-effect models.

\section{DISCUSSION}

To the best of our knowledge, this is the first prospective, multicentre observational cohort study to clarify the efficacy, safety and tolerability of nintedanib in Japanese patients with early-stage IPF classified according to the Japanese IPF disease severity staging classification system.

Several clinical trials of nintedanib in patients with IPF have reported its inhibitory effect on reduced lung function, incidence of acute exacerbation of IPF and worsened health-related QOL. ${ }^{5-7}$ Based on these trials, the use of nintedanib is recommended for the treatment of patients with IPF in the official ATS/ERS/JRS/ALAT clinical practice guidelines ${ }^{3}$ and Japanese IPF treatment guidelines. ${ }^{9}$ However, drug therapy in patients with IPF is not widespread. ${ }^{18}$ Data from Europe and the USA suggest that only approximately $60 \%$ of patients with IPF are receiving nintedanib or pirfenidone. Almost a quarter of the pulmonologists were more concerned about the adverse effects of drug therapy than the risk of disease progression, whereas patients have reported that they wanted more information about the prognosis of their disease and pharmacological treatment options. Furthermore, the patients were more concerned about preventing disease progression than avoiding medicationrelated adverse effects. ${ }^{1920}$ A similar survey was conducted in Japan, and comparable results were reported; $40 \%$ of patients with IPF received nintedanib or pirfenidone, and only $15 \%$ and $34 \%$ of patients with IPF stage I and II received these drugs, respectively. Physicians tend to focus more on symptoms and adverse effects during communication with patients. ${ }^{21}{ }^{22}$ Antifibrotic therapies are associated with several adverse events, including gastrointestinal events. The most common adverse effect of nintedanib is diarrhoea, which was reported in $62.4 \%$ of patients treated with nintedanib compared with $18.4 \%$ of patients who were administered a placebo in the INPULSIS trials. ${ }^{23}$ Although most of the adverse effects of nintedanib are controllable, the risk-benefit balance of nintedanib treatment for patients in the early stage of IPF should be clarified in a real-world setting.

In the present study, we plan to observe patients for 3 years and examine the FVC change, and incidence of and time to adverse events leading to the discontinuation 
and dose reduction of nintedanib. Time to acute exacerbation, death, FVC, DLco decline and changes in serum biomarkers will also be examined. The present study will clarify the efficacy, safety and tolerability of nintedanib for patients with early-stage IPF in the real world. As a result, the risk-benefit balance of nintedanib treatment could be clarified for patients with early stages of IPF.

\section{ETHICS AND DISSEMINATION}

This study will be performed in accordance with the Declaration of Helsinki and Ethical Guidelines for Medical and Health Research Involving Human Subjects. The study protocol and informed consent documents were approved by the Institutional Review Board at Nagasaki University Hospital (approval number 19102146) and each participating site. Written informed consent was obtained from all participants. Patient recruitment has begun and is ongoing. The results will be disseminated through scientific peer-reviewed publications and national and international conferences.

\section{Author affiliations}

${ }^{1}$ Department of Respiratory Medicine, Nagasaki University Graduate School of Biomedical Sciences, Nagasaki, Japan

${ }^{2}$ Research Institute for Diseases of the Chest, Graduate School of Medical Sciences, Kyushu University, Fukuoka, Japan

${ }^{3}$ Division of Respirology, Neurology and Rheumatology, Department of Medicine, Kurume University School of Medicine, Kurume, Japan

${ }^{4}$ Department of Respirology and Clinical Research Institute, National Hospital Organization Kyushu Medical Center, Fukuoka, Japan

${ }^{5}$ Department of Respiratory Medicine, lizuka Hospital, lizuka, Japan

${ }^{6}$ Department of Respiratory Medicine, Kumamoto University Graduate School of

Medical Sciences, Kumamoto, Japan

${ }^{7}$ Department of Respiratory Medicine, Fukuoka University Chikushi Hospital, Chikushino, Japan

${ }^{8}$ Division of Respiratory Medicine, Saiseikai Kumamoto Hospital, Kumamoto, Japan ${ }^{9}$ Innovation Platform \& Office for Precision Medicine, Nagasaki University Graduate School of Biomedical Sciences, Nagasaki, Japan

${ }^{10}$ Clinical Research Center, Nagasaki University Hospital, Nagasaki, Japan

Contributors NS and HM conceived the trial. NHam, MO, KT, HIch, HIsh, KI and NHos initiated and conceptualised the study. SM developed the statistical analysis. All authors contributed to protocol refinement and have read and approved the final version.

Funding This work is supported by the Nippon Boehringer Ingelheim Co. Ltd.

Competing interests NS, NHam, MO, KT, HIch, HIsh, KI and HM received lecture fees from the Nippon Boehringer Ingelheim.

Patient and public involvement Patients and/or the public were not involved in the design, or conduct, or reporting, or dissemination plans of this research.

Patient consent for publication Obtained.

Provenance and peer review Not commissioned; externally peer reviewed.

Open access This is an open access article distributed in accordance with the Creative Commons Attribution Non Commercial (CC BY-NC 4.0) license, which permits others to distribute, remix, adapt, build upon this work non-commercially, and license their derivative works on different terms, provided the original work is properly cited, appropriate credit is given, any changes made indicated, and the use is non-commercial. See: http://creativecommons.org/licenses/by-nc/4.0/.

\section{ORCID iD}

Noriho Sakamoto http://orcid.org/0000-0002-7577-476X

\section{REFERENCES}

1 Natsuizaka M, Chiba H, Kuronuma K, et al. Epidemiologic survey of Japanese patients with idiopathic pulmonary fibrosis and investigation of ethnic differences. Am J Respir Crit Care Med 2014;190:773-9.

2 Kim DS, Collard HR, King TE. Classification and natural history of the idiopathic interstitial pneumonias. Proc Am Thorac Soc 2006;3:285-92.

3 Raghu G, Rochwerg B, Zhang Y, et al. An official ATS/ERS/JRS/ALAT clinical practice guideline: treatment of idiopathic pulmonary fibrosis. An update of the 2011 clinical practice guideline. Am J Respir Crit Care Med 2015;192:e3-19.

4 Homma S, Sugino K, Sakamoto S. Usefulness of a disease severity staging classification system for IPF in Japan: 20 years of experience from empirical evidence to randomized control trial enrollment. Respir Investig 2015;53:7-12.

5 King TE, Bradford WZ, Castro-Bernardini S, et al. A phase 3 trial of pirfenidone in patients with idiopathic pulmonary fibrosis. $N$ Engl $J$ Med 2014;370:2083-92.

6 Richeldi L, du Bois RM, Raghu G, et al. Efficacy and safety of nintedanib in idiopathic pulmonary fibrosis. $N$ Engl $J$ Med 2014;370:2071-82.

7 Crestani B, Huggins JT, Kaye M, et al. Long-term safety and tolerability of nintedanib in patients with idiopathic pulmonary fibrosis: results from the open-label extension study, INPULSIS-ON. Lancet Respir Med 2019;7:60-8.

8 Richeldi L, Cottin V, du Bois RM, et al. Nintedanib in patients with idiopathic pulmonary fibrosis: Combined evidence from the TOMORROW and INPULSIS(®) trials. Respir Med 2016;113:74-9.

9 Homma S, Bando M, Azuma A, et al. Japanese guideline for the treatment of idiopathic pulmonary fibrosis. Respir Investig 2018;56:268-91.

10 Abe M, Tsushima K, Sakayori M, et al. Utility of nintedanib for severe idiopathic pulmonary fibrosis: a single-center retrospective study. Drug Des Devel Ther 2018:12:3369-75.

11 Song JW, Ogura T, Inoue Y, et al. Long-term treatment with nintedanib in Asian patients with idiopathic pulmonary fibrosis: results from INPULSIS $®-O N$. Respirology 2020;25:410-6.

12 Azuma A, Taniguchi H, Inoue $\mathrm{Y}$, et al. Nintedanib in Japanese patients with idiopathic pulmonary fibrosis: a subgroup analysis of the INPULSIS $\otimes$ randomized trials. Respirology 2017;22:750-7.

13 Raghu G, Remy-Jardin M, Myers JL, et al. Diagnosis of idiopathic pulmonary fibrosis. An official ATS/ERS/JRS/ALAT clinical practice guideline. Am J Respir Crit Care Med 2018;198:e44-68.

14 Chiba H, Otsuka M, Takahashi H. Significance of molecular biomarkers in idiopathic pulmonary fibrosis: a mini review. Respir Investig 2018;56:384-91.

15 Ley B, Ryerson CJ, Vittinghoff E, et al. A multidimensional index and staging system for idiopathic pulmonary fibrosis. Ann Intern Med 2012;156:684-91.

16 Collard HR, Ryerson CJ, Corte TJ, et al. Acute exacerbation of idiopathic pulmonary fibrosis. An international Working Group report. Am J Respir Crit Care Med 2016;194:265-75.

17 Raghu G, Collard HR, Egan JJ, et al. An official ATS/ERS/JRS/ALAT statement: idiopathic pulmonary fibrosis: evidence-based guidelines for diagnosis and management. Am J Respir Crit Care Med 2011;183:788-824.

18 Maher TM, Strek ME. Antifibrotic therapy for idiopathic pulmonary fibrosis: time to treat. Respir Res 2019;20:205.

19 Maher TM, Molina-Molina M, Russell A-M, et al. Unmet needs in the treatment of idiopathic pulmonary fibrosis-insights from patient chart review in five European countries. BMC Pulm Med 2017;17:124.

20 Maher TM, Swigris JJ, Kreuter M, et al. Identifying barriers to idiopathic pulmonary fibrosis treatment: a survey of patient and physician views. Respiration 2018;96:514-24.

21 Tomioka H, Kamita M, Azuma H. Barriers to antifibrotic therapy in idiopathic pulmonary fibrosis: a survey of patient and physician views in Japan (Part1). Jpn Open J Respir Med 2020;4:e00097.

22 Tomioka H, Kamita M, Azuma H. Patient-physician communication on treatments for idiopathic pulmonary fibrosis:a survey of patient and physician views in Japan (Part2). Jpn Open J Respir Med 2020;4:e00098.

23 Corte T, Bonella F, Crestani B, et al. Safety, tolerability and appropriate use of nintedanib in idiopathic pulmonary fibrosis. Respir Res 2015;16:116. 